\title{
La familia y políticas públicas
}

\section{The family and public policies}

DOI: http://dx.doi.org/10.17981/cultedusoc.11.1.2020.06

Recibido: 11/12/2019 Aceptado: 14/02/2020. Publicado: 02/03/2020

\section{Mariana Andrea Pinillos Guzmán}

Universidad Santo Tomás. Bogotá, D.C. (Colombia)

marianapinillos@usantotomas.edu.co

Para citar este artículo:

Pinillos, J. (2020). La familia y políticas públicas. Cultura, Educación y Sociedad, 11(1). 88-102. DOI: http://dx.doi.org/10.17981/ cultedusoc.11.1.2020.06

\section{Resumen}

La familia es un factor determinante en la construcción de las políticas públicas, las cuales deben ser formuladas en atención a los intereses y necesidades del grupo familiar. Al respecto, es importante considerar los múltiples e importantes roles que tienen sus miembros, tanto en la formulación como en la concreción de las políticas públicas. Desde este referente, el presente artículo tiene como objetivo comprender la concepción de familia asumida en las políticas públicas del estado colombiano, específicamente al analizar seis políticas públicas, un programa de Estado y el acuerdo de paz, en los cuales la familia hace presencia. El componente metodológico se fundamenta en la sistematización de experiencias, a través del análisis de contenido para establecer relación entre la sintaxis, la semántica y la pragmática, de forma tal que se devele el tratamiento que a la concepción de familia se hace en el ámbito de la política pública. Entre los principales resultados destacan como en la familia recae la responsabilidad de participar activamente en el cambio político y social, a la luz de los condicionantes sociohistóricos. Se concluye en la necesidad de fortalecer la interacción de la familia con el contexto cultural en su lógica de adaptación y mecanismos relaciones con los demás subsistemas sociales.

Palabras clave: Familia; políticas públicas; estructuras; interacciones; dilemas.

\begin{abstract}
The family is a determining factor in the construction of public policies, which must be formulated with the interests and needs of the family group in mind. In this regard, it is important to consider the multiple and important roles that its members have, both in the formulation and in the implementation of public policies. From this point of view, this article aims to understand the concept of family assumed in the public policies of the Colombian state, specifically by analyzing six public policies, a state program and the peace agreement, in which the family is present. The methodological component is based on the systematization of experiences, through the analysis of content to establish a relationship between syntax, semantics and pragmatics, in such a way that the treatment of the concept of family in the sphere of public policy is revealed. Among the main results, it is worth noting that the family is responsible for actively participating in political and social change, in the light of socio-historical conditions. The conclusion is that there is a need to strengthen the interaction of the family with the cultural context in its logic of adaptation and mechanisms for relations with other social subsystems.
\end{abstract}

Keywords: Family; public policies; structures; interactions; dilemmas.

"Este artículo del proyecto de Tesis denominado "Comprensión de la familia como sujeto político" como parte del desarrollo de la Tesis doctoral en Estudios Sociales de la Universidad Externado de Colombia, con apoyo en la formación académica de la Universidad Santo Tomás. 


\section{INTRODUCCIÓN}

La familia es el primer espacio donde se da la socialización, donde ocurre el intercambio de saberes sobre la vida cotidiana. Las relaciones familiares pueden ser positivas si son de apoyo mutuo, respeto y confianza, pero también, pueden ser desfavorables y no apuntar al crecimiento de sus miembros, cuando se presentan situaciones anómalas que derivan de comportamientos inadecuados en alguno de sus miembros. En la familia se generan una red de interacciones, mediada por diversos aspectos que influyen en sus propias dinámicas (Demarchi, Aguirre, Lozano y Viveros, 2015).

En el plano de relación entre familia y políticas públicas, se podría inferir que en el ámbito político la familia ha sido definida desde diferentes lógicas, a partir de lo que representa su participación activa en los múltiples procesos asociados al cambio y transformación sociopolítica y contextual.

Se parte de la premisa que la política pública en relación con la familia actúa como un paisaje adaptativo rugoso en tanto que se convierte en palabras de Reynoso (2011) y Duque (2015) en el espacio de fase o repertorio de soluciones posibles a la complejidad de los problemas de las familias que operan como sistemas adaptativos complejos, desde lógicas co-evolutivas que implican cambios constantes en correspondencia con la necesidad de reconfiguraciones históricas y sociales. En este marco referencial el artículo se orienta a comprender la concepción de familia asumida en las políticas públicas del estado colombiano; específicamente, se analizan seis políticas públicas, un programa de Estado y el acuerdo de paz, en que la familia hace presencia

\section{Metodología}

El artículo se fundamenta en una investigación cualitativa; específicamente se trabaja con un diseño teórico- documental, a través de la técnica del análisis de contenido. La referida técnica permite establecer las conexiones existentes entre el nivel sintáctico del texto y sus referencias semánticas y pragmáticas; es decir, el investigador se pregunta qué significación tiene o qué significa el texto (Martínez, 2013) estableciendo unidades de análisis, focos en común que tienen las políticas y en las que se enmarca el significado, rol y la pragmática de la familia en las mismas.

En este sentido, Marín (2012) señala que el análisis de contenido contribuye a develar los componentes de un texto, con la intención de estudiar sus componentes y las interacciones sistémicas que ocurren entre ellos. El análisis se aplica a la Constitución Política Nacional de Colombia (Asamblea Nacional Constituyente, 1991), el Acuerdo final para la terminación del conflicto y la construcción de una paz Estable y duradera (Alto Comisionado para la Paz, 2016) y el documento sobre la política pública de familias (2012-2022) del Ministerio de Salud y Promoción Social (Ministerio de Salud y Promoción Social, 2012).

El análisis se configura con base a las siguientes categorías: Caracterización de las familias; estucturas, funciones y dinámicas relacionales. 


\section{Resultados y Discusión}

\section{Caracterización de las familias, estructuras, funciones y dinámicas relacionales}

Una primera apuesta que se hace de la familia, y que tiene relación directa con la definición plasmada en la Constitución Política Nacional de Colombia (1991), es la definición de la familia como núcleo fundamental de la sociedad, definición encontrada en la Política de envejecimiento humano y vejez, el acuerdo de paz y la política pública de primera infancia, que aunque no la define de manera explícita como núcleo, si la considera como "el contexto natural para el desarrollo integral de los niños y las niñas de 0 a 6 años" (Ministerio de la Protección Social, Ministerio de Educación Nacional e Instituto Colombiano de Bienestar Familiar, 2007, p. 25); se analiza la definición de contexto natural, como el núcleo, cuya base pretende establecer este ámbito primario en el que se funda la sociedad, fortaleciendo la construcción inicial de los sujetos y asignando responsabilidades a la familia de base en relación con formas de comportamiento de los sujetos en primera infancia.

En este sentido, el acuerdo de paz hace una comprensión de la familia de manera estructural, entendiéndola como grupo familiar compuesto por el o la cónyuge, compañero (a), los hijos menores de edad o quienes estén estudiando hasta los 25 años, a falta de hijos los padres del desmovilizado se consideran parte del grupo familiar, las personas con condiciones de discapacidad son incluidas en el grupo familiar (Alto Comisionado para la Paz, 2016).

Esta comprensión del grupo familiar busca identificar quiénes son los sujetos que pueden definirse como beneficiarios del proceso de desmovilización y a la vez tener conocimiento de la red de apoyo principal que se tienen para el desmovilizado. Además, la familia es considerada, en el Acuerdo de Paz (Alto Comisionado para la Paz, 2016), como un núcleo que puede o no propiciar el cambio social por sus funciones vinculares, en tanto que puede definir la prospectiva vital de quienes están en proceso de reintegración, como afirma el documento:

La familia de los desmovilizados pueden tener dos papeles contradictorios en el proceso de reintegración. Puede ser expulsora y motivar el retorno del desmovilizado a la violencia o puede ser el motor que lo mantiene activo en su ruta de reintegración y el puente de comunicación pacífica con las comunidades receptoras (Departamento Nacional de Política Económica y Social, DNP, 2008, p. 56).

La familia entonces cumple varias funciones a saber: a. mantiene una función evolutiva desde lógicas interacciónales para potencializar el cambio; b. representan un factor determinante en la efectividad del proceso de reintegración social y c. se convierte en un eje donde recae la responsabilidad de un proceso que se enmarca en procesos políticos y sociales. Se pueden entender así los requerimientos que surgen del sistema social hacía la familia como espacio para la formación y monitoreo de cada uno de sus miembros, es decir, su concepción como lugar -objeto- encargada de cumplir con asignaciones sociales, se crea una paradoja sobre la protección de ésta por parte del Estado o la protección de la familia al Estado para garantizar la paz.

Para el 2012 se construye la política pública de familias (2012-2022) en la cual se redefine la idea de familia como núcleo fundamental de la sociedad y se asume como sujeto 
colectivo de derechos. "La familia se considera como un sujeto colectivo de derechos que, a su vez, es garante de los derechos de sus integrantes individualmente considerados. En esta concepción, es agente responsable del ejercicio de los derechos de las personas que conforman el grupo familiar junto con la sociedad y el Estado" (Ministerio de Salud y Promoción Social, 2012, p. 21). Al respecto, se concibe una política pública de familia a en torno a dos actores y beneficiarios que son la familia, como sujeto colectivo y sus integrantes individualmente considerados.

Otras políticas como la política pública de discapacidad definen la familia desde la posibilidad evolutiva que mantiene, reconociéndola como cambiante y participante, así la familia se define como "una unidad social compleja, diversa y plural ubicada en un contexto social, cultural y político; un agente político, corresponsable de la vigencia de los derechos y desarrollo integral de sus integrantes, siendo la mediadora entre las personas que se encuentran bajo su cuidado, las instituciones del Estado y las organizaciones sociales. También es un sujeto colectivo de derechos, cuyas dinámicas desarrollan su capacidad de agencia, es decir su libertad efectiva para alcanzar aquello que valora" (Ministerio de Salud y Promoción Social, 2014a, p. 20).

Se dan aperturas al pensar la familia como "un sujeto colectivo de derechos y garante de los derechos de sus miembros. Es una unidad compleja que media el diálogo entre el sujeto individual, titular en ejercicio de sus derechos en todos los momentos del proceso vital, y el Estado, la sociedad y los demás agentes externos que la interpelan” (Ministerio de Salud y Protección Social citado por Ministerio de Salud y Promoción Social, 2014a, p. 74); la familia al considerarse como sujeto, logra establecer procesos de diferenciación en tanto que, la familia ya no es única y generalizable, sino que se define desde la particularidad de su identidad, así "Cada familia es un grupo de personas que teniendo o no un lazo de sangre, comparte una historia, asigna unos roles a cada uno de sus miembros, establece unas relaciones, se comunica de una forma particular, instaura ciertas normas y tiene un proyecto de vida común. Las relaciones establecidas en ella descansan sobre sentimientos de apego, afecto y lealtad que perduran a través del tiempo (Moreno y Cárdenas citado por Ministerio de Salud y Promoción Social, 2014a, p. 74).

Estos avances en las comprensión de la concepción del constructo familia, posibilita pensar en ella como un sistema adaptativo complejo, donde se establecen procesos coevolutivos y adaptativos de acuerdo a las etapas del cambio histórico- social, se concibe como sujeto dialogante con el Estado; así entonces la familia en las políticas transita de ser un instrumento regulador de la sociedad a ser un sujeto colectivo de derechos que coevoluciona, se vincula, se busca su participación en relación con sus necesidades con la sociedad y el Estado.

Estas particularidades se muestran en las políticas y en los marcos legislativos a través de las transformaciones sociales de la estructura, roles y dinámicas familiares que conducen a la política a crear novedades en el reconocimiento de las familias. Se derivan diversidad en la tipología de las familias en tanto:

La nupcialidad a través del matrimonio perdió su carácter hegemónico. Se introdujeron otras formas de alianzas consensuales, aumentaron los nacimientos extramatrimoniales y las madres solteras. El divorcio abrió las posibilidades de ruptura de las alianzas y la constitución de nuevas parejas (Ministerio de Salud y Promoción Social, 2012, p. 3). 
Con la redefinición de la nupcialidad y la legitimación de nuevas formas de establecer uniones, se suman los procesos migratorios, por las necesidades económicas y por procesos de guerra, esto ha reconfigurado la estructura familiar lo que conduce a nuevas tipologías de familia desde las políticas públicas entre las que se pueden mencionar:

Las familias nucleares constituidas por el padre la madre y sus hijos e hijas, las familias monoparentales conformadas por el padre y sus hijos e hijas o por las madres y sus hijos e hijas, las familias unipersonales, las conformadas por los abuelos y/o abuelas y sus nietos y nietas, las familias de nido vacío, las familias reconstituidas según el modelo nuclear pero con uniones anteriores que conforman familias mixtas desde el punto de vista de los hijos e hijas, las familias sin hijos y sin intención de procrear, las familias trasnacionales, las familias unidas por vínculos de solidaridad o de amistad" (Ministerio de Salud y Promoción Social, 2012, p. 25).

Estos cambios en las estructuras familiares incorporan nuevas dinámicas relacionales convocadas también por los cambios sociales que se han presentado; por ejemplo, con el ingreso de la mujer al mercado laboral la familia se redefine, pues hoy tanto hombres como mujeres se encuentran cumpliendo con las lógicas del sector empleador; esto puede llevar a que la familia sea tratada en un segundo plano; por ello, la política de familia 2012-2022 destaca que "la familia se ocultó y quedó subordinada a los tiempos de la producción. Las mujeres llevan a cabo la gestión familiar en tiempos clandestinos y en sus horas de descanso" (Ministerio de Salud y Promoción Social, 2012, p. 3). Así los cambios sociales llevan a pensar en la flexibilidad del género en contraste de posibilidades, ser cuidador y proveedor no puede estar anclado a condiciones biológicas, sino a procesos de adaptación y coevolución de los sistemas familiares.

Estos cambios en estructuras y funciones en las familias, como respuesta a los procesos adaptativos ante las transformaciones sociales configuradas, son reconocidas en las políticas al establecen algunas aperturas ante nuevas formas de ser y configurar familia. Sin embargo, los cambios creados para la definición de la familia, se establecen en las políticas como una situación transitoria, como consecuentemente Velásquez (2009) plantea:

Un proceso integrador de decisiones, acciones, inacciones, acuerdos e instrumentos, adelantado por autoridades públicas con la participación eventual de los particulares, y encaminado a solucionar o prevenir una situación definida como problemática. La política pública hace parte de un ambiente determinado del cual se nutre y al cual pretende modificar o mantener ( $\mathrm{p}$. 156).

Desde esta danza constante de acoples y nuevas posibilidades, se establecen puntos de bifurcación que se centran en la emergencia de dilemas en las familias, los cuales demandan cambios en la sociedad y en las políticas mismas. Uno de los primeros dilemas donde se pueden anclar varias de las transformaciones que la familia demanda, es el cuidado; este constructo puede ser explicado desde varias perspectivas, a saber: las prácticas de cuidado, la economía del cuidado, el manejo del tiempo; aristas que tienen fuerte conexión con el género en el desempeño del mismo y los cambios sociodemográficos emergentes. 
Las prácticas del cuidado se han configurado como una acción que corresponde al rol de la mujer, esto ha tenido cambios a través de movimientos feministas y de nuevas masculinidades. Sin embargo, el rol de cuidadoras se mantiene y genera duplicación del trabajo de las mujeres como se reporta en la Encuesta del Uso del Tiempo -ENUT-del 2012 (Departamento Administrativo Nacional de Estadística-DANE, 2014), en la cual se muestra cómo las mujeres mantienen una sobre carga laboral dividida entre el trabajo remunerado y el trabajo correspondiente a las prácticas de cuidado y tareas domésticas.

Esto abre el marco de discusión en las políticas públicas sobre a quién corresponde el cuidado y la importancia que debe tener el rol paterno en la sociedad como una posibilidad legitima de construir relaciones nutricias en la familia. Esto posibilita redefinir los roles configurados culturalmente de cuidador y proveedor, para pensar en el trabajo en equipo y la equidad en los roles y de cargas.

Las prácticas de cuidado han correspondido a procesos de crianza y cuidado de los niños, así como el cuidado a los adultos mayores y es en las familias donde se viven estas prácticas que implican una reorganización de todos los miembros en torno a las mismas. El cuidado se convierte en un elemento clave en las políticas pues éste juega un papel que, si bien ha sido instaurado por las dinámicas sociales y culturales, merece ser reconocido como parte de la economía, es decir, implica reconocer acciones de trabajo en la micro economía, o economía de la vida cotidiana, idea que contradice la definición de trabajo decente definido en la política pública del mismo, desde la cual el trabajo decente es denotado como:

Las aspiraciones de la gente durante su vida laboral, significa contar con oportunidades de un trabajo que sea productivo y que produzca un ingreso digno, seguridad en el lugar de trabajo y protección social para las familias nichos, mejores perspectivas de desarrollo personal e integración a la sociedad, libertad para que la gente exprese sus opiniones, organización y participación en las decisiones que afectan sus vidas, e igualdad de oportunidad y trato para todas las mujeres y hombres. Este concepto reconoce el trabajo como fuente de dignidad personal, estabilidad familiar, paz en la comunidad y como punto de convergencia de los siguientes componentes, definidos por la OIT (Ministerio del trabajo, 2017, p. 3).

Si bien el trabajo decente debe proteger la dignidad, la libertad, el tiempo de dedicación y el salario, esto no solo debe reconocer lógicas laborales que implican productividad y se desarrollan en espacio públicos, bajo lógicas del mercado que implican oferta y demanda. El reconocimiento del trabajo en espacios privados promueve el bienestar humano, cubriendo necesidades para quienes son cuidados y las otras personas cercanas. En este sentido la definición de trabajo decente planteada en la política requiere una actualización, desde distinciones que impliquen lógicas colaborativas del cuidado y desde el reconocimiento de éste, cabe retomar la definición mencionada anteriormente acerca de cómo operar en la cotidianidad la libertad, la equidad y el derecho al descanso y al ocio, cuando el cuidado compromete tiempos libres del tiempo laboral reconocido como remunerado.

La economía del cuidado tienen un lugar importante para reconocerse en el mundo laboral y el cuidado cambia en tanto que no solo se ancla a procesos de crianza sino al cuidado de los adultos mayores. Los cambios demográficos que se presenten, muestran que 
el envejecimiento de la población se está dando con rapidez; así lo plantea la política de envejecimiento humano, que retoma estudios realizados por la Comisión Económica para América Latina y el Caribe-CEPAL donde consideran que "en el futuro cercano serán las personas adultas mayores y las personas con dependencia funcional las que constituirán la carga de asistencia mayoritaria" (CEPAL, 2012). Ambas poblaciones mantienen unos altos índices de vulnerabilidad y desigualdad social, que implican tener lógicas de cuidado social que vayan más allá de los microcontextos sociales.

Colombia tiene el más alto porcentaje de personas mayores de 60 años que carecen de ingresos: $42 \%$ de las mujeres y un poco más del 25\% de los hombres (CEPAL, 2012). Simultáneamente, es uno de los países en los que hay menor cubrimiento de la seguridad social en pensiones: menos del $40 \%$ de quienes trabajan están cotizando a la seguridad social en pensiones; y solo cerca del $25 \%$ de las personas adultas mayores recibe algún tipo de pensión, resaltando que más del 70\% de las personas pensionadas reciben una pensión que no supera los dos salarios mínimos mensuales. Al año 2010, el 27\% de los hombres y el 19\% de las mujeres, mayores de 59 años, tienen como principal fuente de ingreso a las pensiones (Dulcey, Arrubla y Sanabria, 2013).

Estas condiciones humanas muestran cómo las familias tienen retos importantes frente a los adultos mayores que implican no solo, redefinirse desde lógicas económicas frente a la manutención de una persona, sino también frente al cuidado de la misma, la familia queda sobrecargada de responsabilidades y el Estado desde sus políticas plantea soluciones para sectores sociales muy pobres que se basan en procesos asistencialistas, más no se cobija a toda la población. Entender el cuidado como la posibilidad de tejer red de solidaridad desde acciones sociales y políticas que se instauren en las políticas públicas es importante, así la familia podría no sobrecargarse en la endogamia del cuidado si se tejieran redes de cuidado social que posibiliten el bienestar. Al respecto, Akl, Pilar y Aponte (2016) destacan la importancia de que en los individuos se generen niveles de bienestar en la medida en que se empoderan del contexto y logran superar situaciones adversas. En este sentido, es importante retomar las recomendaciones planteadas por la CEPAL (2012), donde expone la importancia de transitar hacia la construcción de la dependencia funcional y el cuidado como asuntos de responsabilidad colectiva, que deben ser atendidos mediante prestaciones y servicios para maximizar la autonomía y el bienestar de las familias y los individuos, en el marco de los sistemas de protección social (CEPAL, 2012).

Entonces se devela una paradoja para las familias y es mantener las condiciones nutricias afectivas en el cuidado y la imposibilidad de fallar ante el mismo, aunque los tiempos de trabajo laboral y de cuidado desgasten a quienes cuidan y la penalización impuesta cuando se establecen procesos de desprotección. Cobra importancia otro elemento de la cotidianidad de las familias y que se enmarca en la política de las familias y es el tiempo, más exactamente el uso del tiempo como un dilema presente en los miembros de las familias.

El tiempo de la familia se confunde con el tiempo laboral y el tiempo del descanso la vida familiar carece de tiempos propios, está sujeta a las exigencias del tiempo y el espacio del trabajo; la estructura de la producción es una institución formalizada con horarios fijos, ascensos condicionados al rendimiento según criterios rígidos; los únicos aspectos flexibles son la seguridad en el empleo y los salarios (Ministerio de Salud y Promoción Social, 2012, p. 2) 
Con esto se ve cómo la familia carece de tiempos propios, la flexibilización laboral y el manejo de información conducen a una continua producción del ser humano trascendiendo límites temporales y quitando fronteras espaciales con el otro y a la vez estableciendo dilemas sobre las prioridades de la vida, las obligaciones familiares se administran a través de la tecnología, las relaciones familiares se difuminan entre estar juntos o estar en el trabajo y a se puede crear estrés por la misma saturación de actividades, en especial en las mujeres como lo plasma la política de las familias.

"Estudios realizados por las entidades internacionales, demuestran que esta situación de las mujeres afecta su salud, reduce la productividad y afecta la calidad de vida de los hijos e hijas. La participación comunitaria se atiende en las pausas entre los trabajos anteriores" (Ministerio de Salud y Promoción Social. 2012, p. 3).

El ingreso de la mujer al mercado laboral duplica en trabajo para ellas, por tanto, es importante recobrar las voces de movimientos feministas que buscan equidad en la asignación y reparto de funciones y el manejo del tiempo en el espacio privado. La Encuesta Nacional del Uso del Tiempo -ENUT-muestra una sobrecarga en los tiempos que la mujer emplea en el desarrollo de trabajo - remunerado y no remunerado- frente al uso del tiempo de los hombres (Tabla 1).

TABLA 1.

Participación y tiempo en las actividades de trabajo no comprendido en el sistema de cuentas nacionales por sexo

(Distribución porcentual-hh:mm. Total nacional. Año 2012, 2013 y 2016-2017

\begin{tabular}{|c|c|c|c|c|c|c|c|c|}
\hline \multirow[b]{2}{*}{$\begin{array}{l}\text { Actividades de trabajo no comprendido en el Sistema de } \\
\text { Cuentas Nacionales }\end{array}$} & \multicolumn{4}{|c|}{ Mujeres } & \multicolumn{4}{|c|}{ Hombres } \\
\hline & $\begin{array}{l}\text { Participación } \\
\text { 2012-2013 }\end{array}$ & $\begin{array}{l}\text { Participación } \\
\text { 2016-2017 }\end{array}$ & $\begin{array}{c}\text { Tiempo por } \\
\text { participante } \\
\text { (hh:mm) } \\
\text { 2012-2013 }\end{array}$ & $\begin{array}{c}\text { Tiempo por } \\
\text { participante } \\
\text { (hh:mm) } \\
2016-2017\end{array}$ & $\begin{array}{l}\text { Participación } \\
\text { 2012-2013 }\end{array}$ & $\begin{array}{l}\text { Participación } \\
\text { 2016-2017 }\end{array}$ & $\begin{array}{c}\text { Tiempo por } \\
\text { participante } \\
\text { (hh:mm) } \\
\text { 2012-2013 }\end{array}$ & $\begin{array}{c}\text { Tiempo por } \\
\text { participante } \\
\text { (hh:mm) } \\
\text { 2016-2017 }\end{array}$ \\
\hline Suministro de alimentos a miembros del hogar & $\overline{72,4}$ & $\overline{74,4}$ & 02:09 & $02: 03$ & 22,1 & $\overline{24,9}$ & $00: 56$ & $00: 58$ \\
\hline Mantenimiento de vestuario para las personas del hogar & 40,0 & 38,0 & $01: 18$ & 01:15 & 8,9 ] & 9,1 & $00: 43$ & $00: 44$ \\
\hline Limpieza, mantenimiento y reparación para el hogar & 68,5 & 68,9 & 01:17 & 01:20 & 33,4 & 34,2 & 00:56 & 01:01 \\
\hline Compras y administración para el hogar & 26,8 & 23,6 & 00:50 & $00: 55$ & 22,2 & 20,7 & $00: 49$ & $00: 55$ \\
\hline $\begin{array}{l}\text { Actividades de cuidado con menores de } 5 \text { años } \\
\text { pertenecientes al hogar }\end{array}$ & 16,4 & 16,3 & 01:23 & 01:29 & 10,9 & 10,5 & $01: 14$ & 01:21 \\
\hline Cuidado fisico a miembros del hogar & 22,8 & 20,9 & 01:16 & 01:23 & 4,4 & 3,8 & $00: 38$ & $00: 43$ \\
\hline Apoyo a miembros del hogar & 9,3 【 & 8,6 & 01:17 & 01:22 & 4,3 & 3,6 & 01:02 & 01:09 \\
\hline Voluntariado & 3,4 & 3,0 & $02: 51$ & 03:03 & 1,9 & 1,8 & $02: 35$ & $02: 36$ \\
\hline Cuidado pasivo (estar pendiente) & 43,2 & 35,4 & 05:57 & 07:07 & 23,1 & 16,1 & 03:49 & 05:55 \\
\hline Traslados & 20,1 & 13,6 & $00: 25$ & $00: 26$ & 16,8 & 12,3 & $00: 27$ & $00: 26$ \\
\hline
\end{tabular}

Fuente: DANE (2014).

Dicha distinción en el uso del tiempo recalca la importancia de establecer cambios en los en la distribución de las funciones en los espacios privados, así como el tiempo usado en las mismas. El manejo del tiempo no se ancla solamente al quien se cuida, en tanto que el cuidado se comprende como las acciones de protección, supervisión y ayuda que garantizan la supervivencia de las personas, cuya autonomía e independencia física o cognitiva se carece o se ha perdido (Huenchuan, 2014), implica cómo se cuida a los cuidadores, es decir, cómo quienes cuidan logran establecer procesos de protección a sí mismo en el establecimiento de relaciones equitativas y democráticas, lo cual puede proteger las interacciones de las familia creando cambios ante pautas de violencias que se configuran en escenarios de estrés y cansancio, aunado a la dificultad en llegar a acuerdos equitativos; este ejercicio implica llevar a cabo procesos de conciliación de los tiempos y en la redistribución de funciones que posibiliten el cuidado de la salud física y mental de todos los miembros de las familias. 
Esta división del manejo del tiempo y la borrosidad de los límites en los espacios privados y el contexto laboral mantiene repercusiones importantes en las dinámicas familiares y en la construcción de la salud mental de los individuos. El tercer elemento que cobra vigencia para la definición de la familia y los dilemas emergentes que se articulan con los dos elementos anteriormente expuestos refiere procesos de calidad de vida para la familia. Característica que se describen en las diferentes políticas, situaciones particulares de cómo viven las familias.

La calidad de vida se concibe como la sensación de bienestar que tiene una persona o grupo de personas frente a sus condiciones económica, sociales, de salud y protección social que dan la posibilidad a las personas de establecer procesos de evolución y desarrollo desde la dignidad. La calidad de vida entonces se considera como un entramado de recursos provenientes del contexto que posibilitan o no el bienestar de las personas. En Colombia acorde con la política pública de familias (2012-2022), se mantienen diferencias sustanciales entre el contexto urbano y el rural frente a la infraestructura y el acceso de servicios públicos, encontrando que en las ciudades el 99\% de las viviendas cuentan con servicio de energía y en el campo el $91 \%$. El servicio de acueducto público llega a $90.8 \%$ de las viviendas en las zonas urbanas y al 17\% en las zonas rurales y el de alcantarillado llega al 92\% de las viviendas en la zona urbana y al $22 \%$ en las zonas rurales. El gas es el combustible más usado en las ciudades, en las zonas rurales es la leña (Ministerio de Salud y Promoción Social, 2012).

Las mismas diferencias pueden encontrarse en el acceso a la educación la asistencia escolar de la población entre 6 y 15 años es en la zona urbana del 96\% y en la rural del 93\%. De 16 a 24 años, solo 10 jóvenes de las zonas rurales están en el sistema educativo y 30 en las áreas urbanas. El mayor nivel de deserción se presenta en los grados cuarto y quinto, siendo dicha deserción tres veces mayor en la zona rural que en la urbana.

La desigualdad en Colombia tiene un coeficiente de GINI de 0,52, siendo éste uno de los países más desiguales de la región con evidencia en la infraestructura de las viviendas, servicios de salud, educación y recreación, que en zonas rurales son muy precarios y en algunas inexistentes. Además de estos factores, en Colombia hay otra diferencia importante entre el contexto urbano y el rural, tiene relación con los procesos de violencia desatados por el mismo que marcan el bienestar de las personas en tanto que determinan los lazos vinculares que tienen y la posibilidad de cuidarlo o de vivir en su fragmentación.

A estas características poblacionales, las políticas agregan los cambios demográficos que se están presentando y que implican cambios en la compresión de la familia. En primer lugar, se encuentra el aumento progresivo en la tasa de envejecimiento del país; la política de vejez lo expone así

Se puede establecer que el proceso de transición demográfica que manifiesta Colombia evidencia cambios progresivos experimentados por los diferentes grupos de edad de la población. En efecto, hacia 1950 la población colombiana menor de 5 años representaba un 18\%, en tanto que la de 60 años y más tan sólo llegaba a un 5\%. En el año 2000, estos dos subgrupos representan un 11 y un $7 \%$ respectivamente. Es significativa entonces la pérdida de peso relativo de los menores y el incremento de las personas de mayor edad. Al año 2015, Colombia registra un total de 48.203.405 habitantes, de los cuales el $11.0 \%$ son mayores de 60 años [en este orden de ideas] La proporción de personas de 60 a 74 años cuyo peso relativo se incrementó en $4.5 \%$ en los pasados 50 años pasará a representar un 14\% en el año 2050. 
Por su parte, el grupo de 75 años o más, que corresponde a las personas de mayor edad, con más altos riesgos y problemas más complejos en el campo sociosanitario y en muchos aspectos de su vida personal y familiar, se incrementará en forma acelerada llegando a representar más de un 7.5\% en el año 2050" (Ministerio de Salud y Promoción Social, 2014b, p. 8)

Estos cambios socio demográficos, se convierten en un reto para las familias, la sociedad y el estado, en tanto que al tener mayor tasa poblacional de adultos mayores se gestan nuevas necesidades económicas, políticas, sociales, laborales y de cuidado, todos los anteriores factores repercuten en las dinámicas familiares, en la estructura de las familias y en la necesidad de pensar en el entramado social la construcción de redes solidarias de cuidado que prioricen el rol del adulto mayor.

Otro reto importante a rescatar en las políticas públicas y que compete a las familias y a la sociedad para dar calidad de vida, es la posibilidad de reconocer y dar lugar a las personas con discapacidad, pensar en esto implica generar posibilidades laborales y dar respeto a las mismas; en Colombia 61 de cada 100 personas con condición de discapacidad no recibe ningún tipo de ingresos mientras que, aproximadamente, 30 de cada 100 recibe menos de $\$ 500.000$ mensuales, lo cual muestra como la desde los escenarios laborales se invisibilizan las necesidades de quienes tienen una condición de discapacidad, quedando limitada la posibilidad de tener autonomía e independencia se pone en riesgo, entra y las posibilidades y recursos de las personas y a la vez de las familias. Esto crea una paradoja ante disposiciones jurídicas establecidas referidas a la eliminación de la interdicción en Colombia, se coloca entonces en procesos de mayor vulnerabilidad a las personas en situación de discapacidad, pues no se les reconoce en sus capacidades laborales, pero tampoco se les da lugar a ser protegidos jurídica y económicamente (Ministerio de Salud y Promoción Social, 2014a).

Las políticas, en especial la política de salud mental del 2018, reconoce las características poblacionales en torno a la salud y la enfermedad mental, retomando la encuesta de salud mental realizada en el 2015 por el Ministerio de Protección Social, donde se muestran las cifras de prevalencia en enfermedad mental en Colombia. Partiendo de lo anterior se retoma la definición de la política de salud metal que dan desde la percepción que los colombianos tienen de la misma, consideran así que "la salud mental de los colombianos está en relación con tener una adecuada salud física, comer, dormir y descansar bien, así como también disfrutar de tranquilidad, paz y poder ser capaces de superar las dificultades de la vida diaria" (Ministerio de Salud y Promoción Social, 2018, p. 9). Esta definición tiene una fuerte relación con la calidad de vida; implica que para tener una buena salud mental es importante tener satisfechas las necesidades básicas del ser humano, así como emocionales y afectivas teniendo una correlación directa con la calidad de vida como categoría que da cuenta del bienestar de las personas y las familias; Además de lo anterior, la salud mental mantiene una relación alta con el apoyo social frente a dificultades o problemas, es decir, la capacidad y posibilidad de las personas de tejer redes sociales que creen relaciones de apoyo con diferentes niveles de vinculación: familiares, amigos, vecinos u otras personas.

El Informe regional sobre los sistemas de salud en América Latina y el Caribe (Organización Mundial de la Salud-OMS, 2013), menciona que las personas con trastornos mentales presentan tasas elevadas de discapacidad y mortalidad. Para el caso Colombiano, la depresión mayor unipolar, los trastornos mentales y del comportamiento debidos al uso de 
alcohol, los trastornos de ansiedad y la epilepsia se encuentran entre las primeras veinte causas por Años Vividos con Discapacidad AVD, lo cual afecta a la familia y la pone en un lugar de responsable sobre la vida de aquella persona que se encuentra sintomática. Siendo esta una realidad del país vale la pena pensar en cómo la política puede ofrecer en una voz dialogante con el Estado, posibilidades de pensar la cotidianidad en una calidad de vida, pues si bien los síntomas mencionados hacen parte de la cotidianidad, es importante retomar el cómo los mismos pueden aparecer ante situaciones de no contención en sistemas macrosociales.

En este sentido aparece en la política de salud mental enunciado otro problema que habla de la sociedad misma y es el suicidio La Organización Mundial de la Salud-OMS estima que "la frecuencia del intento de suicidio es 20 veces mayor que la del suicidio consumado" (Boletín de salud mental citado por Ministerio de Salud y "Promoción Social, 2018, p. 11). En Colombia, de acuerdo con el con Sistema Integral de Información de la Protección SocialSISPRO, se muestra un incremento substancial en la tasa nacional del intento de suicidio, siendo en el 2009 de 1,8 por 100.000 habitantes al 2017, de 52,4 por 100.000 habitantes. Así entonces se puede pensar que el suicidio habla de la liquidez de los vínculos existentes en el entramado social, y la configuración relacional dada desde las lógicas del consumo sobre los procesos humanos del ser (Baumman, 2017). Cabe entonces preguntar por la posibilidad que la política pública crea de establecer un tejido social protector y vinculante del ser humano; así la política debería buscar espacios en los que las posibilidades de crear lazos de confianza en las comunidades y redes relacionales permanezcan; establecer articulaciones con otros sistemas y no solo con el de salud para poder pensar que el entramado de cuidado por el otro y por la vida se gesta en las sociedades desde la cotidianidad y desde la articulación intersectorial como una apuesta que atraviesa procesos macrosociales e impactan a los procesos microsociales.

La violencia en la vida cotidiana, también se encuentra inmersa en los procesos de pensar las características de la sociedad y su impacto en la familia, según el Instituto Nacional de Medicina Legal y Ciencias Forenses (2017) para el año 2017 se perdieron 337.36 años de vida saludable, indicador dado por las muerte prematuras generadas por factores relacionados con la violencia.

La violencia en la sociedad ha sido reconocida como un mecanismo simbólico de justicia y reconocimiento que posibilita mediar conflictos y obtener poder, siendo ésta legitimada en la sociedad desde sistemas de creencias, rituales sociales y mitos que posibilitan la instauración y el arraigo en las distinciones de género, capital, valores, cohesión social y cultura. "Dentro de esta última, las normas sociales, prejuicios, estereotipos, creencias e imaginarios justifican, validan y reproducen el uso de la violencia, la ruptura en el tejido social, la baja solidaridad ciudadana y la confianza interpersonal" (Ministerio de Salud y Promoción Social, 2018, p. 16).

La violencia intrafamiliar, se puede comprender como un proceso isomórfico, emergente e interaccional dentro de los microsistemas sociales, que producen y reproducen a la sociedad misma. La política pública de Salud Mental 2018 retoma el estudio de Rivero y Sánchez, publicado en el 2004 por el Centro de Estudios para el Desarrollo Económico (CEDE) de la Universidad de los Andes, el cual confluye: 
La violencia intrafamiliar impacta negativamente los ingresos laborales de las mujeres, reduciendo su participación en el mercado laboral y limitando las opciones de encontrar un empleo satisfactorio. De igual forma, afecta la salud y el rendimiento escolar de los niños y las niñas" (Ministerio de Salud y Promoción Social, 2018, p. 18).

La Encuesta Nacional de Salud Mental (2015) exploró la presencia de eventos traumáticos en la población, producidos por algún tipo de violencia, o por situaciones sumamente estresantes, encontrando que, el 11,7\% de la población infantil (7 a 11 años), el 29.3\% de los adolescentes y alrededor del $41 \%$ de los adultos ha estado expuesto a maltrato físico, abuso sexual, accidentes de tránsito, robo o atraco, ataque de un animal, conflicto armado, enfermedad grave o potencialmente mortal, haber sido testigo de serias lesiones o fallecimiento inesperado de los padres, cuidadores u otro ser querido y el desplazamiento forzado. Se considera que estos eventos tienen afectaciones importantes la salud mental de las personas en Colombia, además de hablar de cómo la sociedad Colombiana está siendo estructurada a través de la imposición del más fuerte sin importar las lógicas de solidaridad, de tal manera que las lógicas relacionales individualistas que dan cumplimento al sistema capital que lleva al olvido de lo humano configura la sociedad y establece procesos de deslegitimación del otro.

\section{Conclusiones}

Como ideas conclusivas del análisis realizado para comprender a la familia, su estructura y dinámicas a través de las políticas públicas, se exponen las siguientes.

Una primera conclusión que puede darse de este recorrido se da en la importancia de reconocer un concepto de familia, pensada como un sistema vivo, cambiante, evolutivo y adaptativo, es decir, un sistema complejo adaptativo que es cambiante y a la vez participante con la biosfera en la que se encuentra inmerso y así interactúa con ésta.

En este sentido, es importante reconocer la interacción de la familia con el contexto cultural en su lógica de adaptación y cambios que pueden establecer de manera relacional, en esta medida es importante que las políticas públicas se reconozcan en su postura y apuesta a intervenir reconociendo las posiciones de todas de manera holística y a la vez local.

Reconocer la familia como un sistema o sujeto participante e interactuante con la sociedad y el estado, posibilita establecer lógicas de comportamiento fractal ante las necesidades de las familias, sin embargo, esta apuesta implica dejar de ver a la familia desde la carencia o características de los miembros, para pensarla en formas articuladas de adaptación y legitimación del otro, esto puede llevar a tener escenarios de democratización familiar y social.

Por tanto, al reconocer la familia como un sistema adaptativo cambiante que interactúa con el estado y la sociedad, las políticas se encuentran en un reto de ser actualizadas histórica y contextualmente y a la vez ser pensadas en tiempos largos de cambios sociales; lo que implica para las políticas establecer nuevas versiones de sí mismas pensadas en contextos, no solo desde las necesidades sino desde las posibilidades de cambio y ajuste que implican los cambios sociales. Así entonces un reto que existe para las políticas es la posibilidad de pensarse en sentidos amplios e interactuantes incluyentes entre sí, más que fragmentadas en poblaciones, temáticas o necesidades. 
Es importante entonces que la construcción de las políticas tenga puentes de conexión que impliquen el reconocimiento de individualidades en las colectividades y recobre la colectividad en sus necesidades, en su historia, en su contexto y las posibilidades de cambio, apropiándose de manera crítica sobre los intereses de los gobiernos.

\section{REFERENCIAS}

Akl, P., Pilar, E. y Aponte, F. (2016). Estrategias de afrontamiento en mujeres víctimas de violencia intrafamiliar. Cultura Educación y Sociedad, 7(2), 105-112. Disponible en https://revistascientificas.cuc.edu.co/culturaeducacionysociedad/article/view/1105/ pdf_238

Bauman, Z. (2017). Amor líquido. Acerca de la fragilidad de los vínculos. México. D.F.: Fondo de Cultura Económica.

CEPAL. (2012). Envejecimiento, solidaridad y protección social: La hora de avanzar hacia la igualdad. Santiago de Chile: ONU.

Demarchi, G., Aguirre, M., Lozano, N. y Viveros, E. (2016). Sobre la dinámica familiar. Revisión documental. Cultura Educación y Sociedad, 6(2), 117-138. Recuperado de https://revistascientificas.cuc.edu.co/culturaeducacionysociedad/article/view/1049

Dulcey, E., Arrubla, D. J. y Sanabria, P. (2013). Envejecimiento y vejez en Colombia: estudio a profundidad. Bogotá, D.C.: Profamilia. Recuperado de https:/fiapam.org/ wp-content/uploads/2014/03/3-ENVEJECIMIENTO-Y-VEJEZ-EN-COLOMBIA.pdf

Duque, R. E. (2015). La investigación como biosfera autoorganizada Diálogos entre psicología clínica, ciencias de la complejidad y estética de los mundos posibles. [Tesis de doctorado]. Pontificia Universidad Javeriana, Bogotá, D.C.

Huenchuan, S. (2014). “¿Qué más puedo esperar a mi edad?” Cuidado, derechos de las personas mayores y obligaciones del Estado. En, S. Huenchuan y R. I. Rodríguez. Autonomía y dignidad en la vejez: Teoría y práctica en políticas de derechos de las personas mayores. México, D.F.: ONU. Recuperado de https://repositorio.cepal.org/ bitstream/handle/11362/39570/S1421014_es.pdf?sequence=1\&isAllowed=y

Marín, F. (2012). Investigación científica. Una visión integrada e interdisciplinaria. Zulia: Ediciones del Vicerrectorado Académico de la Universidad del Zulia. Disponible en https://www.researchgate.net/publication/321547221_Investigacion_cientifica_Vision_integrada_e_interdisciplinaria

Martínez, M. (2013). Ciencia y arte en la metodología cualitativa. (1 ed.). México, D.F.: Trillas.

OMS. (2013). WHO-AIMS: Informe regional sobre los sistemas de salud mental en américa latina y el caribe. [Online]. Disponible en http://www.paho.org/per/images/stories/FtPage/2013/WHO-AIMS.pdf

República de Colombia. Alto Comisionado para la Paz. (2016). Acuerdo final para la terminación del conflicto y la construcción de una paz Estable y duradera. [Online]. Recuperado de http://www.altocomisionadoparalapaz.gov.co/procesos-y-conversaciones/ Paginas/Texto-completo-del-Acuerdo-Final-para-la-Terminacion-del-conflicto.aspx

República de Colombia. DANE. (2014). Encuesta del Uso del Tiempo del 2012. [Online]. Recuperado de http://microdatos.dane.gov.co/index.php/catalog/214/study-description 
República de Colombia. Asamblea Nacional Constituyente. (1991). Constitución política de Colombia [Const.] Artículo 13 [Titulo II]. (2 ed.). Bogotá, D.C.: Legis. Recuperado de http://www.secretariasenado.gov.co/senado/basedoc/constitucion_politica_1991. html

República de Colombia. DNP. (2008). Política Nacional de reintegración social y económica para personas y grupos armados ilegales. [Documento Conpes 3554]. Recuperado de: https://www.reincorporacion.gov.co/es/la-reintegracion/centro-de-documentacion/Documentos/Documento\%20Conpes\%203554\%201\%20Pol\%C3\%ADtica\%20 nacional\%20de\%20reintegraci\%C3\%B3n\%20social\%20y\%20econ\%C3\%B3mica $\% 20$ para\%20personas\%20y\%20grupos\%20armados\%20ilegales.pdf

República de Colombia. Instituto Nacional de Medicina Legal y Ciencias Forenses. (2017). Forensis datos para la vida. [Online]. Recuperado de https://aprendiendoaserpapaz. redpapaz.org/wp-content/uploads/2018/08/Forensis-2017-pdf-interactivo.compressed. pdf

República de Colombia. Ministerio de la Protección Social, Ministerio de Educación Nacional y Instituto Colombiano de Bienestar Familiar. (2007). Política pública de primera infancia, Colombia por la primera infancia. Colombia. [Online]. Recuperado de: https://icbf.gov.co/cargues/avance/docs/conpes_dnp_0109_2007.htm

República de Colombia. Ministerio de Salud y Promoción Social. (2018). Política Nacional de Salud Mental. [Online]. Recuperado de: https://www.minsalud.gov.co/Normatividad_Nuevo/Resoluci\%C3\%B3n\%20No.\%204886\%20de\%202018.pdf

República de Colombia. Ministerio de Salud y Promoción Social. (2014a). Política Pública Nacional de Discapacidad e Inclusión Social 2013-2022. [Online]. Recuperado de: https://www.minsalud.gov.co/proteccionsocial/promocion-social/Discapacidad/Paginas/politica-publica.aspx

República de Colombia. Ministerio de Salud y Promoción Social. (2014b). Política Colombiana de envejecimiento y vejez 2015-2024. [Online]. Recuperado de: https://www. minsalud.gov.co/sites/rid/Lists/BibliotecaDigital/RIDE/DE/PS/Pol\%C3\%ADtica-colombiana-envejecimiento-humano-vejez-2015-2024.pdf

República de Colombia. Ministerio de Salud y Promoción Social. (2012). Política pública nacional para las familias colombianas 2012-2022. [Online]. Recuperado de: http:// www2.congreso.gob.pe/sicr/cendocbib/con4_uibd.nsf/CBA649DA5EBF221205257BF 1007B8494/\$FILE/APolitica_Publica_Familias_Colombianas_2012_2022_(1).pdf

Reynoso, C. (2011). Redes sociales y complejidad: Modelos interdisciplinarios en la gestión sostenible de la sociedad y la cultura. Buenos Aires: SB.

Velásquez, R. (2009). Hacia una nueva definición del concepto "política pública. Desafios, (20), 149-187. Recuperado de https://pdfs.semanticscholar.org/cd36/ecca238ab278d09a96aaf7500a79952e5350.pdf 